\title{
3D surface pressure measurement with single light-field camera and pressure-sensitive paint
}

DOI:

10.1007/s00348-018-2534-z

\section{Document Version}

Accepted author manuscript

Link to publication record in Manchester Research Explorer

\section{Citation for published version (APA):}

Shi, S., Xu, S., Zhao, Z., Niu, X., \& Quinn, M. (2018). 3D surface pressure measurement with single light-field camera and pressure-sensitive paint. Experiments in Fluids. https://doi.org/10.1007/s00348-018-2534-z

\section{Published in:}

Experiments in Fluids

\section{Citing this paper}

Please note that where the full-text provided on Manchester Research Explorer is the Author Accepted Manuscript or Proof version this may differ from the final Published version. If citing, it is advised that you check and use the publisher's definitive version.

\section{General rights}

Copyright and moral rights for the publications made accessible in the Research Explorer are retained by the authors and/or other copyright owners and it is a condition of accessing publications that users recognise and abide by the legal requirements associated with these rights.

\section{Takedown policy}

If you believe that this document breaches copyright please refer to the University of Manchester's Takedown Procedures [http://man.ac.uk/04Y6Bo] or contact uml.scholarlycommunications@manchester.ac.uk providing relevant details, so we can investigate your claim.

\section{OPEN ACCESS}




\title{
3D Surface Pressure Measurement with Single Light-Field Camera and Pressure-Sensitive Paint
}

\author{
Shengxian $\mathrm{Shi}^{{ }^{*}}$, Shengming $\mathrm{Xu}^{1}$, Zhou Zhao ${ }^{1}$, Xiaofu Niu², Mark Kenneth Quinn ${ }^{3^{*}}$ \\ ${ }^{1}$ School of Mechanical Engineering, Shanghai Jiao Tong University, 200240, Shanghai, China \\ 2 School of Electronic Information and Electrical Engineering, Shanghai Jiao Tong University, \\ 200240, Shanghai, China \\ ${ }^{3}$ School of Mechanical, Aerospace \& Civil Engineering, University of Manchester, Manchester, \\ M13 9PL, UK
}

\begin{abstract}
A novel technique that simultaneously measures three-dimensional model geometry, as well as surface pressure distribution, with single camera is demonstrated in this study. The technique takes the advantage of light-field photography which can capture three-dimensional information with single light-field camera, and combines it with the intensity-based pressure-sensitive paint method. The proposed single camera light-field three-dimensional pressure measurement technique (LF-3DPSP) utilises a similar hardware setup to the traditional two-dimensional pressure measurement technique, with exception that the wind-on, wind-off and model geometry images are captured via an in-house-constructed light-field camera. The proposed LF3DPSP technique was validated with a Mach 5 flared cone model test. Results show that the technique is capable of measuring three-dimensional geometry with high accuracy for relatively large curvature models, and the pressure results compare well with the Schlieren tests, analytical calculations, and numerical simulations.
\end{abstract}

Keywords: light-field camera; PSP; 3D surface pressure measurement; depth estimation

*Corresponding author: kirinshi@sjtu.edu.cn (Shengxian Shi)

mark.quinn@manchester.ac.uk (Mark Kenneth Quinn) 


\section{Introduction}

As a non-intrusive, high spatial resolution, surface pressure measurement technique, pressuresensitive paint (PSP) has been widely applied in aerodynamic experimental studies since late 1980s (Kavandi et al. 1990). Unlike the traditional pressure tap and transducer techniques, which only provide point-wise pressure measurements, PSP is capable of measuring the pressure distribution over complex model surfaces. The technique makes use of oxygen quenching of luminescent molecules suitably imaged, with resolution theoretically only limited by the photodetector (Bell et al. 2001, Liu et al. 2005). PSP is capable of measuring both steady and unsteady pressure fluctuations by utilising suitable PSP substrates (or binder materials) and imaging devices (Gregory et al. 2008, Gregory et al. 2014, Nabavi 2010). With continuous efforts being paid to mitigate uncertainties introduced by paint temperature sensitivity, model displacement and deformation, instability of illumination sources, and photo-degradation, the technique has been refined over the past three decades. In addition to better accuracy for lowspeed flow and higher response frequency for high-speed flow measurements, it is of great interest to the aerodynamic measurement community to obtain the pressure distribution over an entire model surface, which normally has complex, three-dimensional geometries.

Measuring the pressure over the complex geometry of a full aerodynamic model is traditionally accomplished by multi-camera configurations. For instance, Engler et al. (2001) measured the surface pressure distribution on an AerMacchi M-346 Advanced Trainer Aircraft model in a transonic wind tunnel. Measurements were made, not just on the aircraft main body, but also pressure distribution over the flaps, air brakes, rudders and ailerons. Pressure signals were captured by eight CCD cameras and mapped onto a three-dimensional grid to produce completesurface pressure data. A different study conducted at the ARA transonic wind tunnel utilised 12 cameras and 16 lamps to measure the pressure distribution around the SAAB Gripen (Vardaki et al. 2012). Multiple-view PSP techniques necessitate the installation, alignment and calibration of several cameras as well as opening multiple optical windows, which are cumbersome for aerodynamic tests, especially for large wind tunnel experiments. This study aims to remove this restriction by capturing the surface geometry and the PSP data simultaneously with a single camera.

Recent progress in light-field photography offers a very promising alternative for multi-camera three-dimensional PSP. The terminology "light field" represents a collection of light rays that 
travel freely in three-dimensional space. The light field is normally modelled as a five-dimensional plenoptic function $L=L(x, y, z, \theta, \varnothing)$, in which $L$ is the radiance density of light rays, $(x, y, z)$ and $(\theta, \varnothing)$ represent the spatial position and angular location of the rays (Levoy 2006). If one needs to capture the light field of an object's surface, for instance, luminescence emitted from a PSP model surface, the plenoptic function can be simplified as $L=L(u, v, s, t)$, where $(u, v)$ and $(s, t)$ are the location that ray intersects with two parallel planes (Levoy et al. 1996, Gortler et al. 1996). To record such a four-dimensional light field, $\mathrm{Ng}$ et al. (2005) proposed a camera architecture that positions a micro-lens array (MLA) one focal length away from the image sensor (termed as light-field camera thereafter). By doing so, any ray of the light field can be determined by its intersection with main lens aperture plane and MLA plane of the light-field camera. It was later demonstrated that a single light-field camera can faithfully capture the three-dimensional light rays of a scene, and a set of new images that are artificially focused at different depths or viewed from different perspectives can be calculated from single raw light-field image ( $\mathrm{Ng} 2006$ ).

By integrating the light-field photography with particle image velocimetry (PIV), this novel singlecamera, three-dimensional imaging technique has been successfully applied to experimental fluid mechanics recently (Ding et al. 2015, Fahringer et al. 2015). In the light-field particle image velocimetry technique (LF-PIV), light-field images of the seeding particles (diameter ranging from $1 \mu \mathrm{m}$ to $50 \mu \mathrm{m}$ ) are captured by a double-exposure light-field camera, which is constructed by precisely assembling a customised MLA with a high-resolution PIV camera (Shi et al. 2016). Two three-dimensional particle image volumes can be reconstructed by either the refocusing method (Fahringer et al. 2015), or a ray tracing method in conjunction with a multiplicative algebraic reconstruction algorithm (Shi et al. 2017). From two consecutively recorded light-field images, the three-dimensional velocity field is then calculated by using a cross-correlation approach. Inspired by the successful application of LF-PIV to complex flow measurement (Li et al. 2017, Xu et al. 2017), the authors propose that the pressure distribution and three-dimensional geometry of a model surface can be simultaneously acquired with single camera by combining light-field imaging with PSP. In this paper, the LF-3DPSP technique will be detailed in Section 2, the demonstration in the hypersonic wind tunnel and related measurement results will be presented in Section 3. Lastly, Section 4 concludes the main findings of current work. 


\section{The Light-Field 3D PSP Technique}

Based around the intensity-based pressure measurement method, the LF-3DPSP technique consists of two parts, which are luminescence light-field capturing and pressure estimation (Figure 1 (a)) and model three-dimensional surface light-field reconstruction (Figure 1 (b)). As Figure 1 shows, LF-3DPSP has similar hardware configuration to a traditional 2D-PSP system for the pressure estimation. However, both the wind-on and wind-off excitation images are captured by a light-field camera instead of conventional CCD or CMOS camera. Furthermore, LF-3DPSP has an additional step for three-dimensional model geometry measurement, which is achieved by recording a light-field image of the model after the wind-off image is taken. In the following subsections, the light-field rendering and reconstruction algorithms will be presented, detailing how the pressure signals and model geometry can be retrieved from light-field images. An overview of the image processing steps in LF-3DPSP is listed in Figure 2.
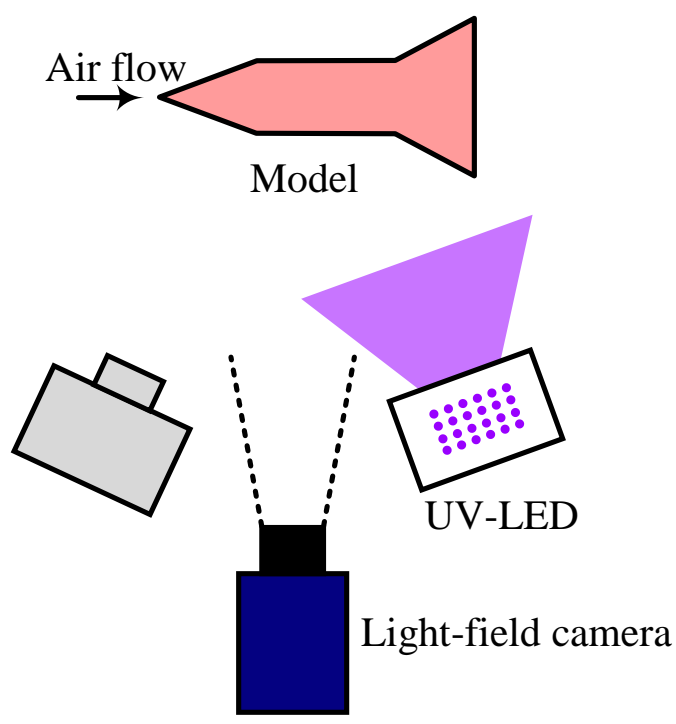

(a)
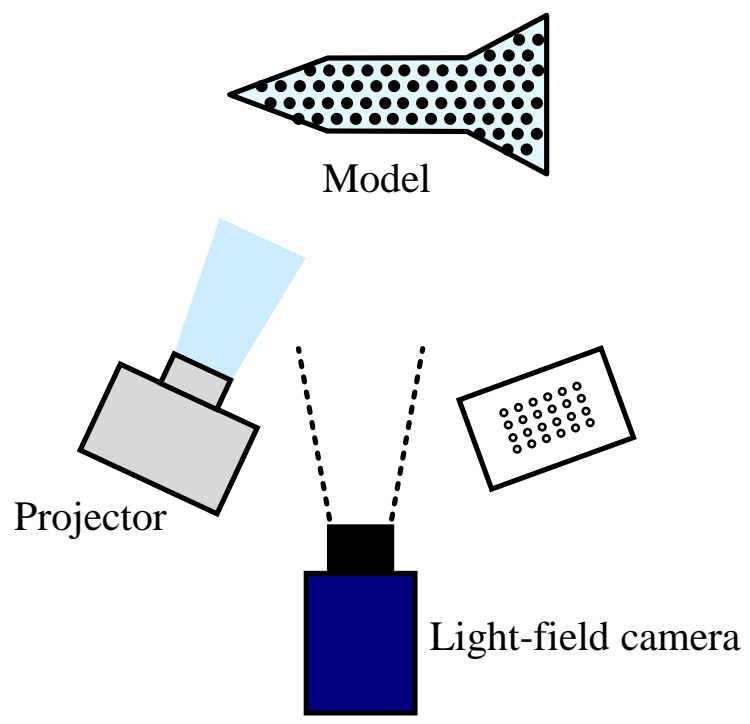

(b)

Figure 1 - Concept of the LF-3DPSP technique: (a) luminescence light-field capturing and pressure estimation and (b) model three-dimensional surface light-field reconstruction

\subsection{Luminescence Light-Field Capturing and Pressure Estimation}

According to the intensity-based method, pressure signals can be derived from the ratio of windon and -off images (Liu et al. 2005) using a modified version of the Stern-Volmer equation given below, where $I$ is the luminescent intensity recorded by the camera, $P$ is the pressure on the 
surface, $A$ and $B$ are calibration coefficients which are functions of temperature ( $r e f$ denotes a reference or wind-off condition).

$$
\frac{I_{\text {ref }}}{I}=A(T)+B(T) \frac{P}{P_{\text {ref }}}
$$

Before taking the image ratio, the raw light-field wind-on and -off images are rendered with lightfield perspective shift algorithm, the procedures of which are outlined in Figure 2 (left-hand side). The first step of light-field image rendering is to determine the centre coordinate of each lenslet ("MLA calibration", Figure 2). This is achieved by taking an image of a white plate with the main lens $\mathrm{f}_{\#}$ set to maximum ( $\mathrm{Ng} 2006$ ). The MLA calibration image appears as an array of white dots on a black background (Figure $3(\mathrm{a})$ ), with each white dot representing a lenslet centre. To determine the coordinate for each lenslet centre, a $3 \times 3$ Gaussian fit is used to achieve a sub-pixel accuracy (red asterisk in Figure 3 (b)). With the availability of the MLA lenslet centre coordinate, the correspondence between each lenslet and the pixel group beneath it can be established.

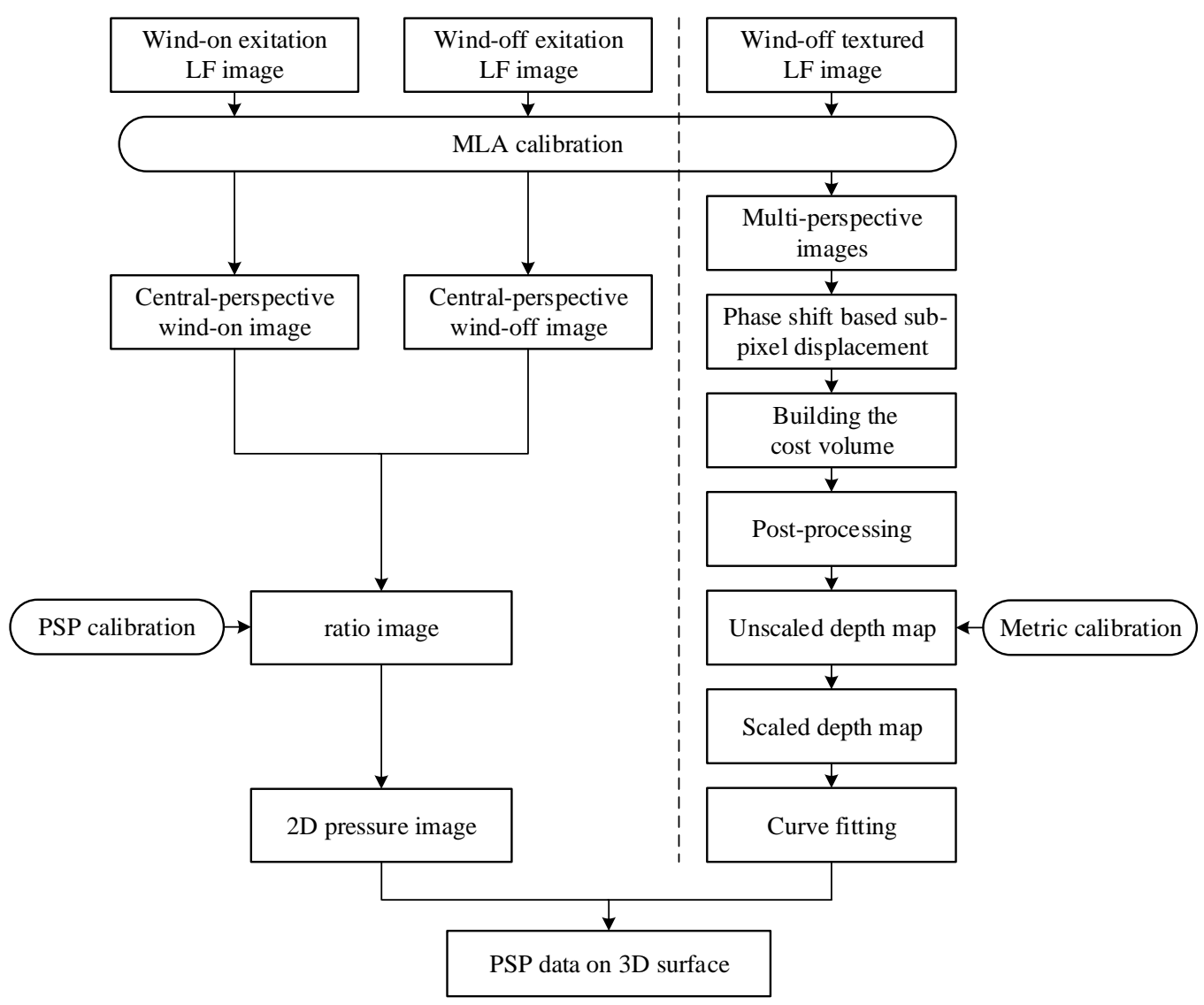

Figure 2 - Flow chart of the image processing steps for the LF-3DPSP technique 


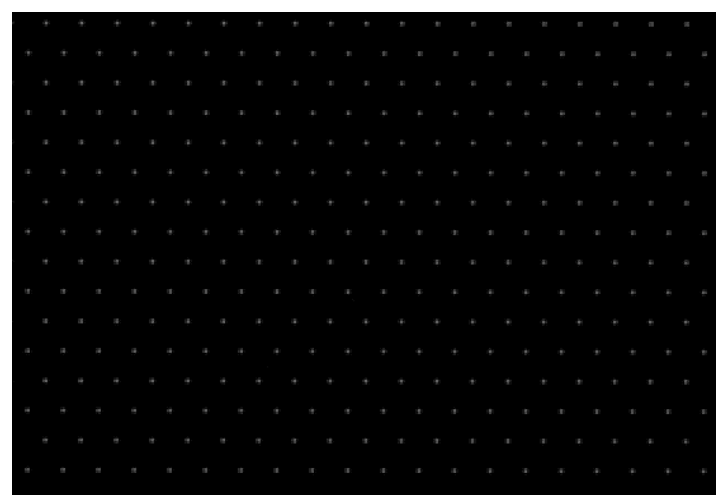

(a)

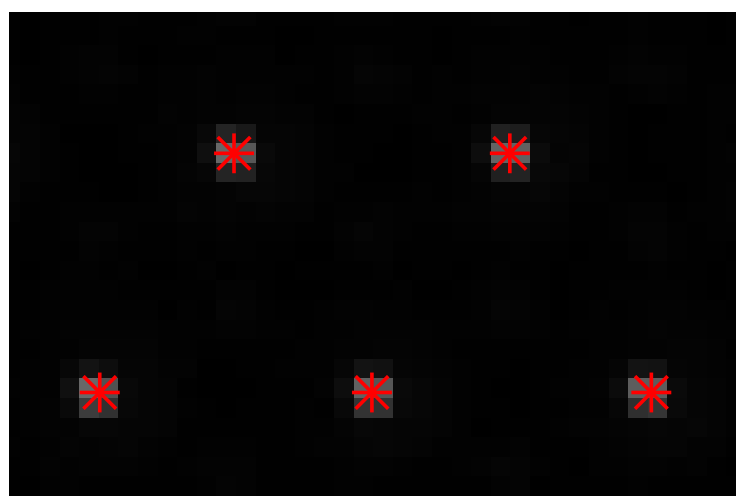

(b)

Figure 3 - Example of (a) MLA calibration image and (b) calculated lenslet centre

For the light-field camera specified in this paper, the CCD/CMOS sensor is located one focal length $\left(f_{M L A}\right)$ behind the MLA (Figure $\left.4(\mathrm{a})\right)$. The angular resolution is estimated using the number of pixels beneath each lenslet (Ng 2006, Georgeiv et al. 2006). Figure 4 demonstrates the one-dimensional schematic of such a light-field camera. While real light-field cameras normally use a very highresolution image sensor and MLA, for simplicity, the schematic shown here only demonstrates 5 lenslets ( $5 \times 5$ in $2 \mathrm{D}$ case) with only 5 pixels ( $5 \times 5$ in $2 \mathrm{D}$ case) behind each micro-lens unit. This arrangement means that $5 \times 5$ new perspective images can be generated from one raw light-field image, which is achieved by selecting and merging a specific pixel from each lenslet. For example, if we select the third pixel (i.e. red pixels in Figure 4 (a) and (b)) from each lenslet and stich them together, we would be able to generate a new image that artificially views the object from the central perspective (the red image in Figure 4 (c)). Similarly, if we select the fifth pixels (i.e. blue pixels in Figure 4 (a) and (b)) for each lenslet, we would generate the upper-most perspective image (the blue image in Figure 4 (c)). For the pressure estimation, only the central perspective of the wind-on and -off light-field images are used ("Central-perspective image", Figure 2). It is possible to use all perspective images for pressure calculation, doing so would require compensating model offset between different perspectives, which is likely to introduce additional errors. As lenslets normally have circular apertures (i.e. the grey circle in Figure 4 (b)), it is not possible to generate all of the $5 \times 5$ perspectives as some outer most pixels are not covered by the microlens unit. Note that the exact number of effective perspectives varies with specific light-field cameras. Information about the in-house light-field camera used in this study will be provided in section 3. For greater details of perspective shift processing, readers are referred to $\mathrm{Ng}$ (2006). 


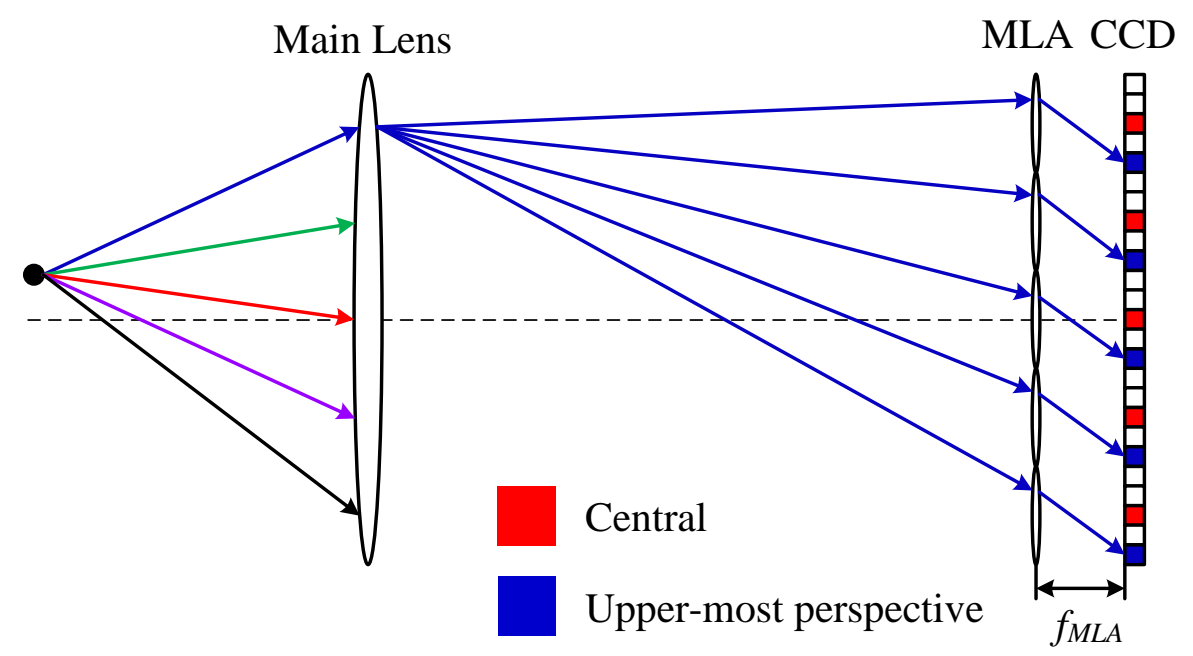

(a)

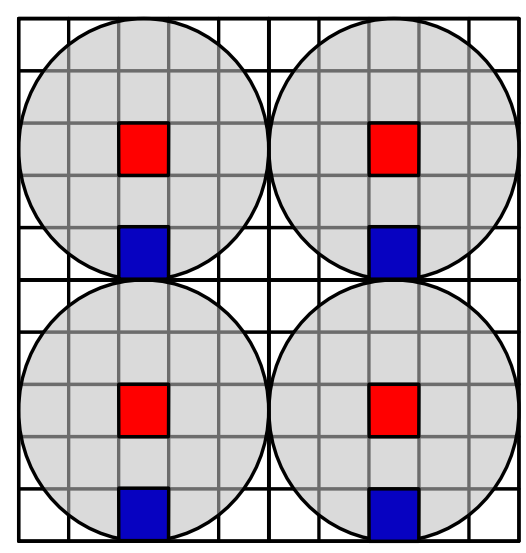

(b)

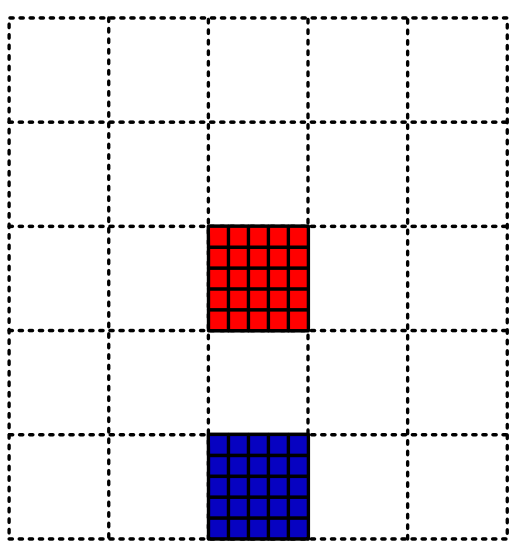

(c)

Figure 4 - Principle of the light-field perspective shift algorithm (a) 1D schematic of the lightfield camera, (b) sub-image of lenslet (showing only four lenslets here) and (c) artificially generated new perspective images (showing only the central and upper-most perspectives)

\subsection{Model Three-Dimensional Surface Light-Field Reconstruction}

Ideally, the three-dimensional information of the model surface could be derived from the windon or wind-off light-field image. However, it was found that the depth estimation from either wind-on or wind-off light-field image is unreliable as the pressure-sensitive paint makes the model surface too featureless to be correctly processed by any depth estimation algorithm. For better three-dimensional estimation accuracy, an extra depth measurement step was included after taking the wind-off light-field image. As Figure 1 (b) shows, a projector was used to project a dense black dot array on a white background onto the model surface when the wind tunnel is off ("Wind- 
off textured LF image", Figure 2). By using such dot array, texture was artificially created on the model surface. The textured light-field image was then processed using a depth estimation algorithm (Jeon et al. 2015). While details about the depth estimation can be found in the reference, the main steps are outlined here for completeness.

To implement the depth estimation, different perspective images (e.g. $5 \times 5$ new perspective images) are firstly extracted from the raw textured light-field image by using the perspective-shift method. Every new perspective image (e.g. the red and pink dot images in Figure 5 (a)), except the central one (e.g. the green dot image in Figure $5(a)$ ), is then shifted with a series of sub-pixel values. For example, the red dot image is shifted 64 different sub-pixel displacements (Figure 5 (b)), or termed as 64 labels by using image interpolation, which will generate for example 64 shifted images for each perspective image, i.e. each shifted image corresponds to a specific label ("Sub-pixel shifting", Figure 2). All of the shifted images (e.g. 64 labels $\times 24$ perspectives) are compared with the central perspective image in terms of pixel gradient and pixel intensity, so as to generate a series of disparity images (e.g. the red dot image is shifted and compared with the green dot image, Figure 5 (b)). These images are grouped according to their corresponding label, and the cost value is calculated by summing up images under the same label ("Building cost volume", Figure 2). For instance, adding all of the 24 disparity images which correspond to the first label would then produce the cost value for that label. Repeating the calculation for all labels produces 64 cost values (or termed as cost volume) for each pixel in the central perspective image (Figure 5 (c)). The depth information of each pixel is finally determined according to the least cost value ("Unscaled depth map", Figure 2). Such a discrete label-based method is commonly used in computer vision to determine the disparities between images. The term "cost volume" refers to a three dimensional space $C(x, y$, label), in which " $x$ " and " $y$ " are the location of a pixel, "label" represents the discrete candidates of disparity value (Figure $5(b))$ and " $C$ " records the difference between an image pattern and local images with different disparity values (Figure 5 (c)). A lower cost value usually indicates a better matching between image pattern and the local image. The depth information calculated using such method only measures the depth of a pixel relative to the light-field camera focal plane (unscaled depth map). The final, scaled depth map is obtained by converting the magnitude of disparity into real depth in millimetres via an in-house metric calibration method detailed below. 


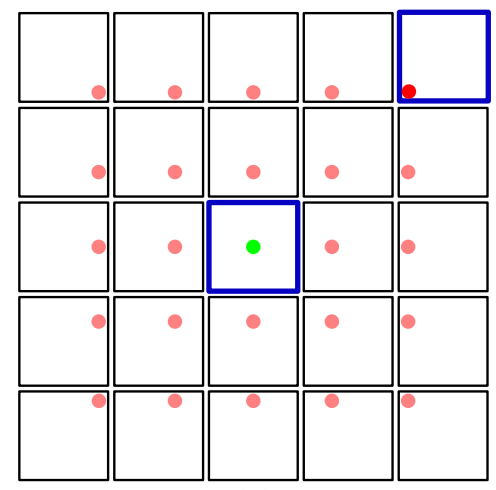

(a)

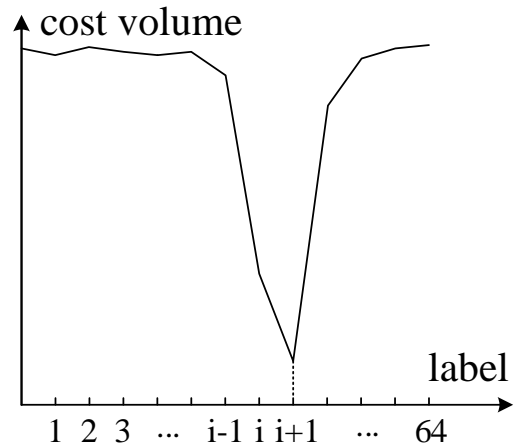

(c)

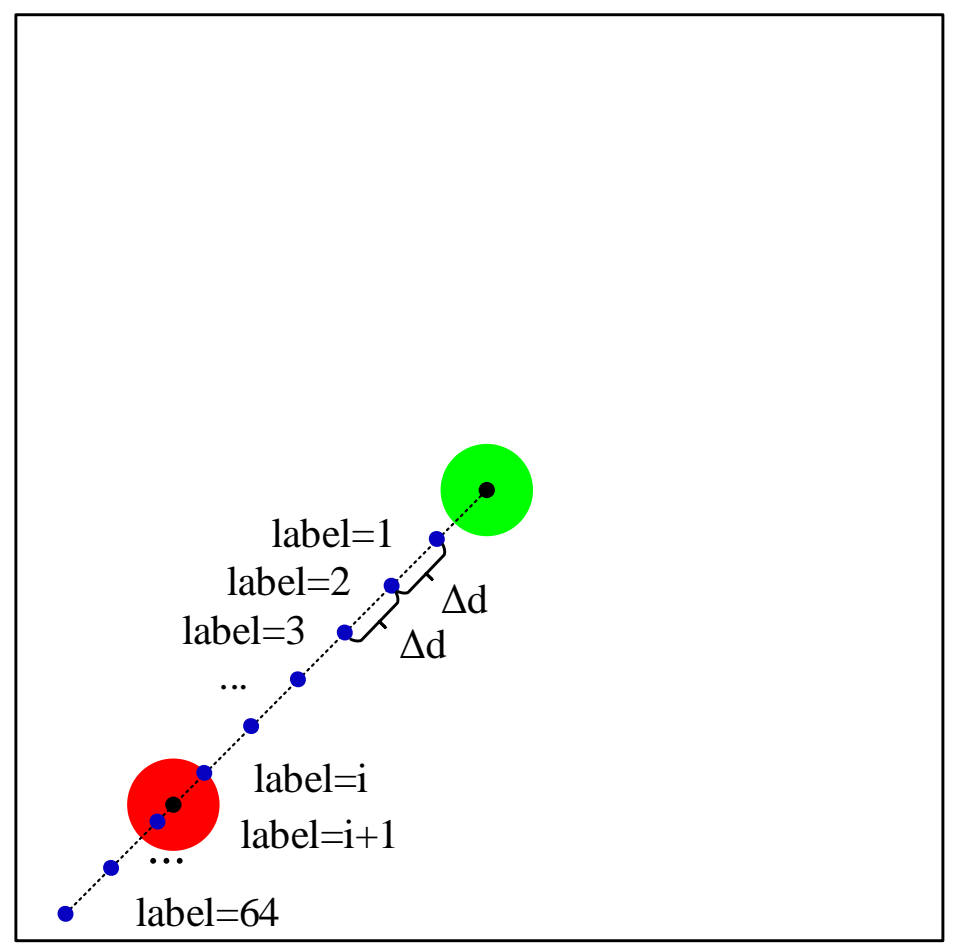

(b)

Figure 5 - Schematic of the depth estimation method (a) $5 \times 5$ perspective images, model is presented as a dot, (b) sub-pixel shifting with different labels and (c) relation between cost volume and label index

The general schematic of metric calibration is shown in Figure 6 ("Metric calibration", Figure 2), which includes a calibration board (detailed in Figure 6 (b)), a high-precision motorised translation system (Thorlabs LNR50S/M, resolution $0.1 \mu \mathrm{m}$ ) and the light-field camera. The calibration board is incrementally translated in one direction with a fixed step size. For each position, images are taken (Figure 6 (c)) and the disparity maps are calculated with the above method. In total there are 20 images captured and processed for 20 steps, the calculated disparity maps and known depth interval are then fitted using a spline fit. This fit is then used for calibrating the unscaled depth map and producing the final depth location in mm unit ("Scaled depth map", Figure 2). As the depth information is given at different labels, the scaled depth map contains information only at discrete locations. To produce a depth map for a continuous full-model surface, the discrete depth data is interpolated by spline curve fitting for each cross-section along $\mathrm{x}$-axis (Figure 7 (b)) of the model ("Curve fitting", Figure 2). 


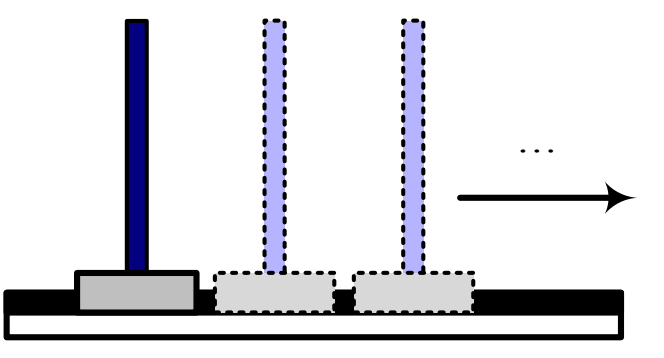

(a)

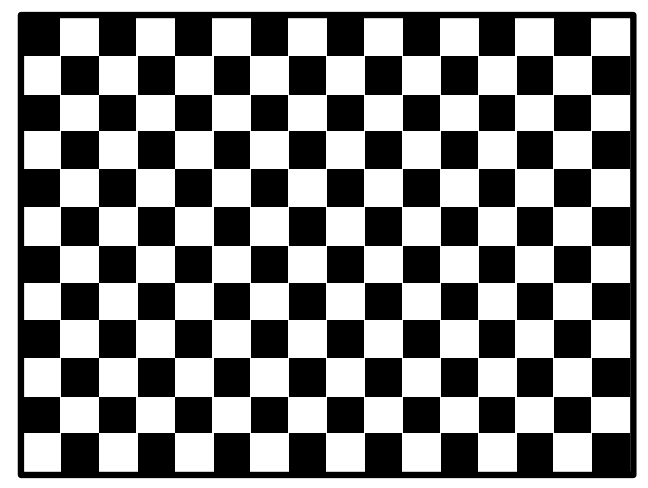

(b)

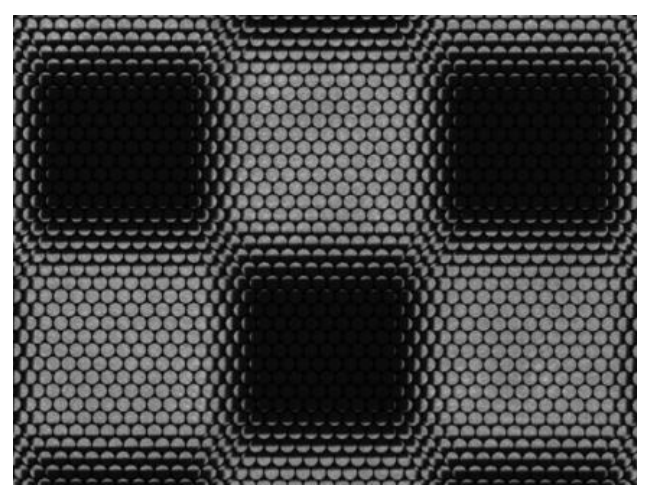

(c)

Figure 6 - (a) Schematic of light-field camera metric calibration, (b) calibration board and (c) light-field image of the calibration board (magnified)

\section{Three-Dimensional Pressure Measurement of a Flared Cone Model in Mach 5 Flow}

To demonstrate the capability of the proposed LF-3DPSP technique, tests were conducted in the intermittent blow-down high supersonic tunnel (HSST) at the School of Mechanical, Aerospace and Civil Engineering at the University of Manchester as used by Erdem et al. (2010, 2011b). The tunnel layout, shown in Figure 7 (a), consists of a free jet expanding into an evacuated, quiescent plenum chamber. The facility is capable of running at Mach 4, 5 and 6; however, the current experiments were performed at Mach 5. With a maximum total pressure of 8.1 bar and large storage tanks, the facility is capable of providing stable run times of 7.5 seconds. The flow is temperature controlled using a resistive heater connected to a high-current lab power supply and is capable of generating total temperatures from 288-950K. For this test campaign, the total temperature was held constant at $300 \mathrm{~K}$ to ensure repeatability. The test section and plenum chamber have multiple quartz windows to enable Schlieren and PSP measurement techniques in the facility. For comparison, Schlieren data was captured using a standard Z-style Töepler system 
with illumination coming from a Newport-Oriel 1kW Xenon Arc Lamp. A vertical knife edge was used to cut off light to a Nikon D5000 SLR camera used as the Schlieren imaging device.

The PSP system consists of ISSI UniFIB PSP paint excited using an in-house-constructed UV LED lamp as was used by Quinn et al. (2017). The PSP was excited using two lamps, one mounted inside the plenum chamber of the wind tunnel and one mounted externally on a tripod to give the most uniform illumination possible. The calibration chamber is also the one used in the study by Quinn et al. (2017) and was used to systematically vary the pressure and temperature independently to generate a calibration response function. The model used during this campaign is the same as was used by Erdem et al. (2011 a, b, c) and is shown in Figure 7 (b). This model was chosen due to the large pressure changes present on the model as well as its large, distributed radii.
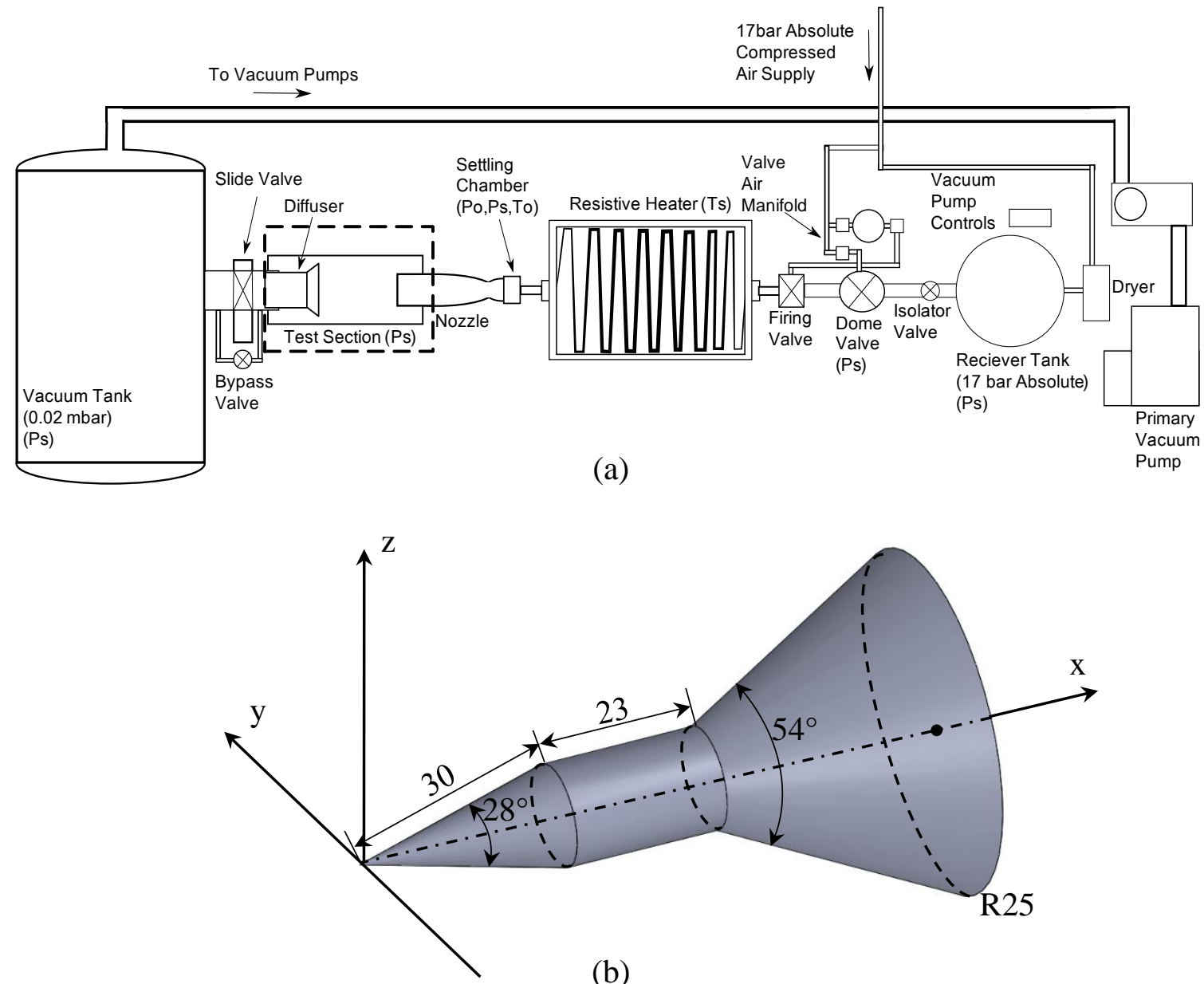

Figure 7 - a) Layout of the hypersonic wind tunnel and (b) geometrical details of the flared cone model (in $\mathrm{mm}$ ) 

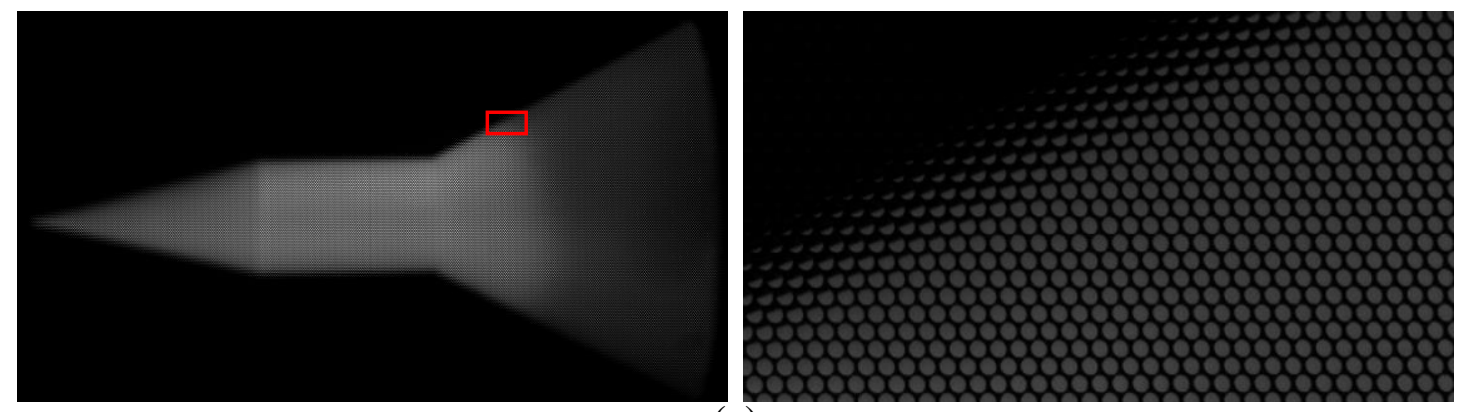

(a)
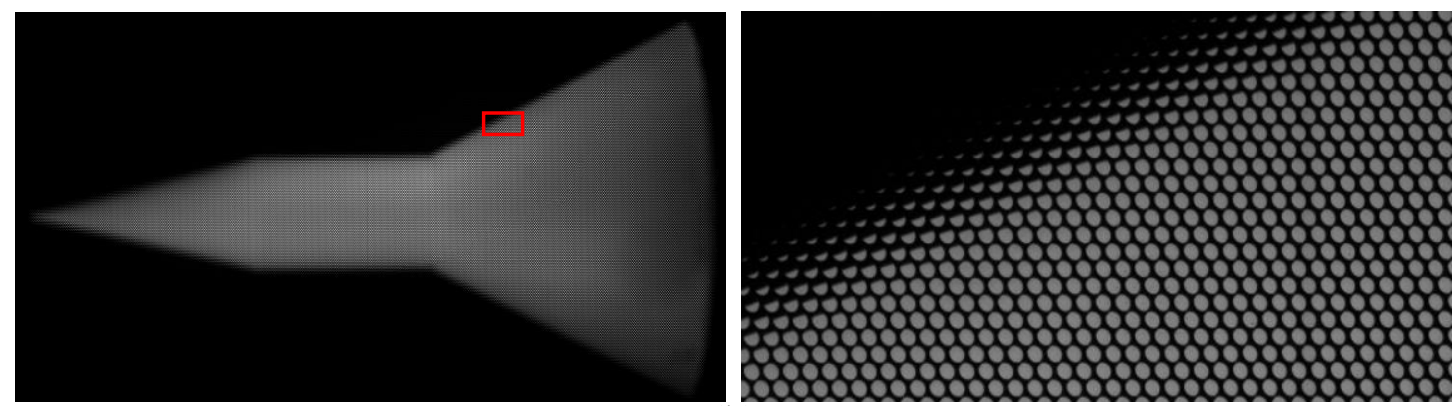

(b)
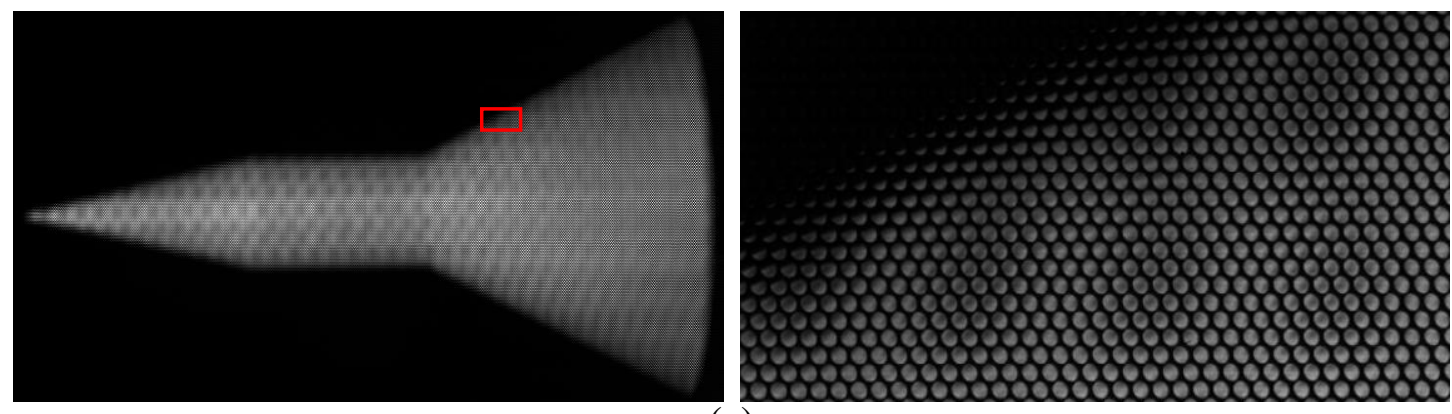

(c)

Figure 8 - Examples of (a) wind-on excitation light-field image and its zoom-in detail, (b) windoff excitation image and its zoom-in detail and (c) textured light-field image and its zoom-in detail for the flared cone model

The wind-on, wind-off PSP images and wind-off textured image were captured by an in-houseconstructed light-field camera with a Micro-NIKKOR $200 \mathrm{~mm}$ lens and a $610 \mathrm{~nm}$ long-pass filter (Galvoptics) in order to separate out the PSP emission and excitation signals. The light-field camera was modified from an Imperx B6640 camera ( $6600 \times 4400$ pixel), which is equipped with a KAI-29050 scientific-grade CCD sensor that offers 12 -bit resolution. A $408 \times 314$ customised hexagonal packing MLA was positioned one focal length $\left(f_{M L A}=308 \mu m\right)$ away from the CCD sensor. For more details about the in-house light-field camera, refer to Shi et al. (2016). An example of 
the captured wind-on, -off and textured raw light-field images is shown in Figure 8, highlighting the sub-image formed beneath each microlens unit.

Following the procedures detailed in Figure 2, the raw light-field images were firstly rendered to generate a series of different perspective images with a resolution of $800 \times 523$. The in-house lightfield camera has $14 \times 14$ pixels beneath each lenslet, which can generate $14 \times 14$ perspective images. In the current experiments, only the central $5 \times 5$ perspective images were used for further processing. In the second step, the two-dimensional pressure image was calculated in a similar fashion as traditional 2D-PSP, that is to take the ratio between the centre perspective of the rendered wind-on and -off excitation images.

In the third step (as detailed in left-hand side, Figure 2 ), the central $5 \times 5$ perspective images generated from the wind-off textured light-field image were used to calculate the scaled depth map in the presence of metric calibration. Figure 9 shows the original depth data along with the fitted curve for three cross sections $x=89.5 \mathrm{~mm}, x=47.0 \mathrm{~mm}$ and $x=24.5 \mathrm{~mm}$. This curve fitting was performed at every cross section along $x$-axis meaning the three-dimensional geometry of the model surface can be obtained, as shown in Figure 10 (a). As the models were manufactured by high-precision CNC, with a machine error of only $\pm 20 \mu \mathrm{m}$, the design geometry was used as ground truth for calculating the absolute depth estimation error. As Figure 10 (b) shows, the measurement errors in section $x=0 \sim 60 \mathrm{~mm}$ are less than $1 \mathrm{~mm}$, proving the ability of the current method in measuring a three-dimensional geometry for a relatively small curvature surface. In this region, the diameter of the model cross section is relatively large such that enough dot images are scattered on the model surface, and hence is suitable for accurate depth estimation. However, with the decreasing model diameter near the model leading edge, there are effectively fewer dot images. Near the tip, there is either a purely white background or black dot image. Without sufficient texture, the current method failed to calculate valid depth data. For small curvature surfaces, current depth measurement accuracy is lower than the photogrametric model deformation measurement methods (Liu et al. 2012). 


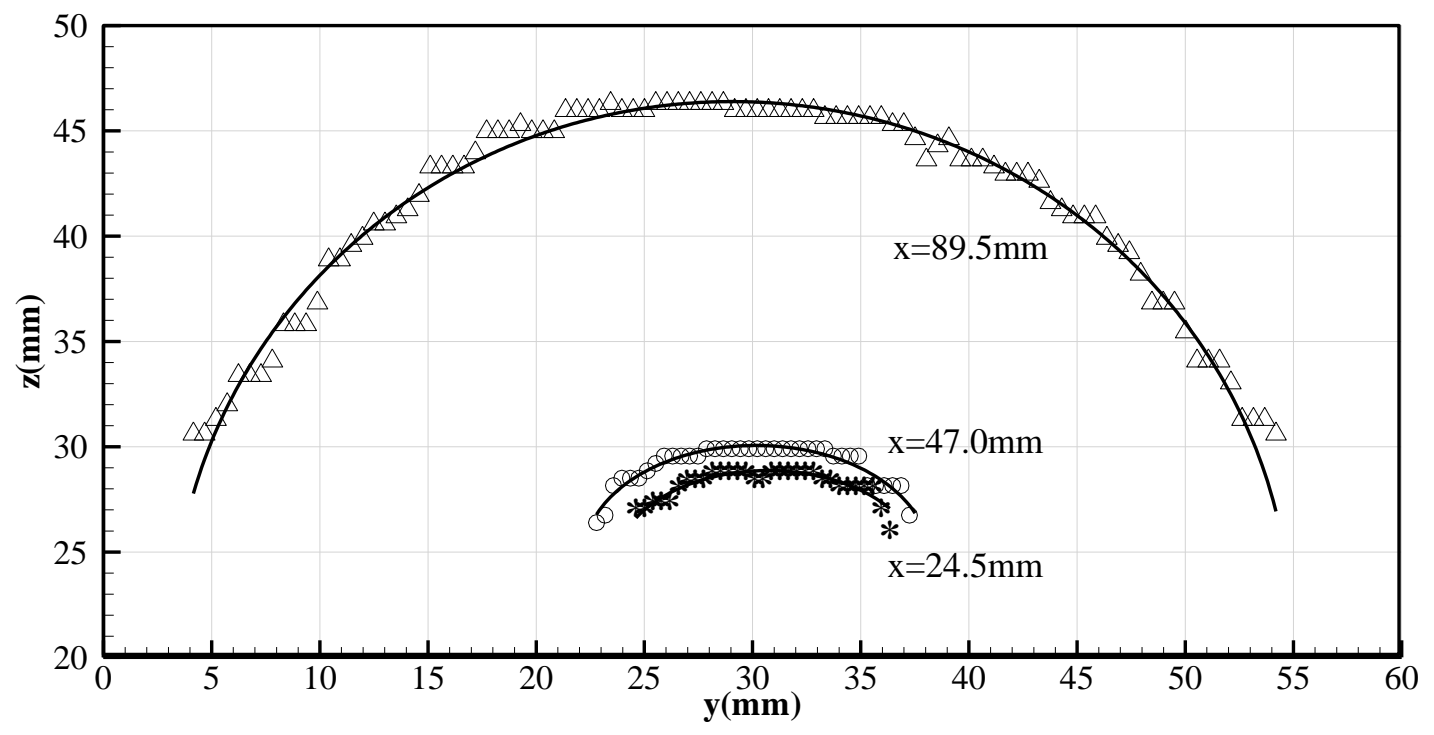

Figure 9 - Measured depth data (denoted as symbols) and fitted curve (denoted as curves) for the flared cone model at three cross sections at $x=89.5 \mathrm{~mm}, x=47.0 \mathrm{~mm}$ and $x=24.5 \mathrm{~mm}$ 


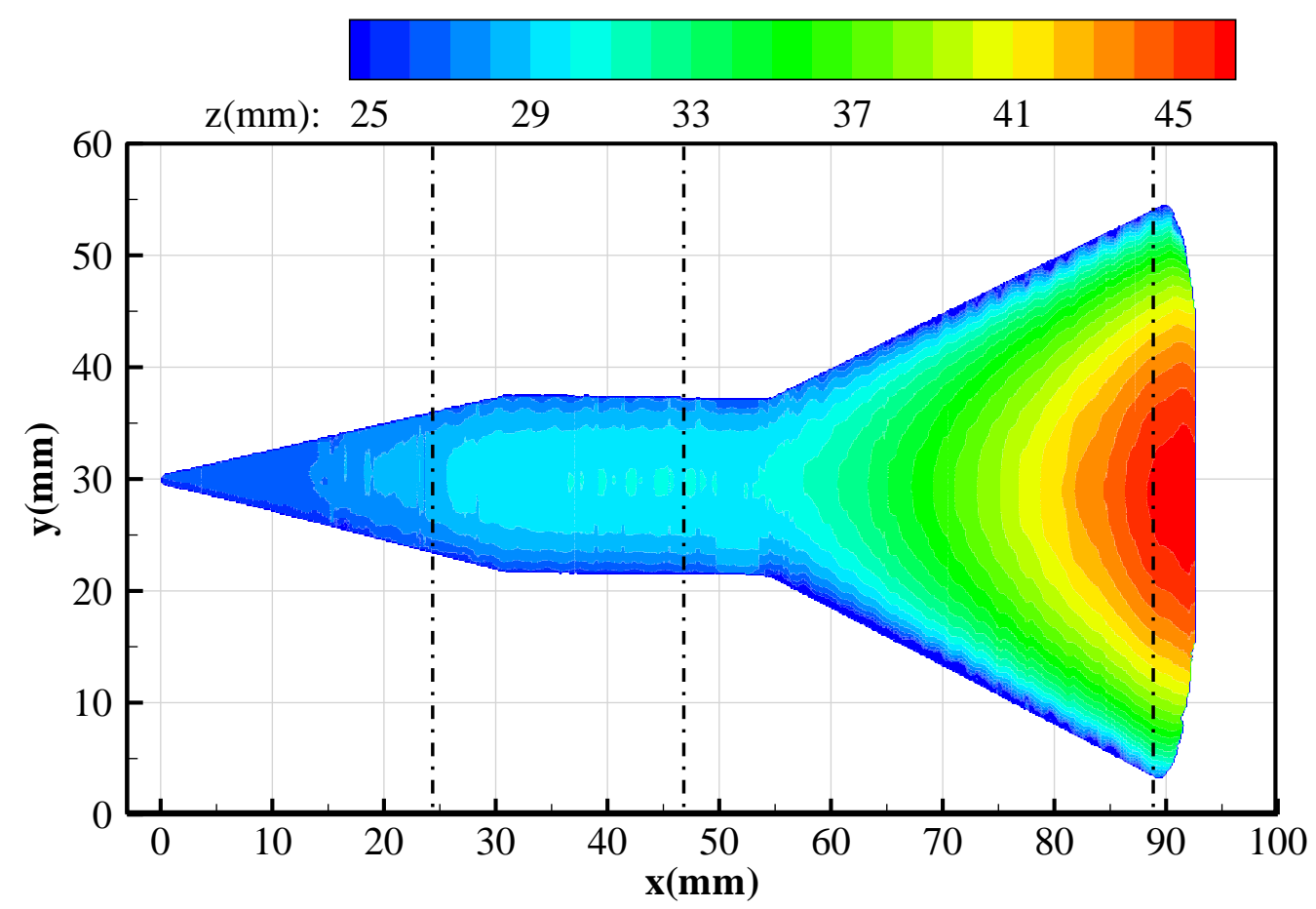

(a)

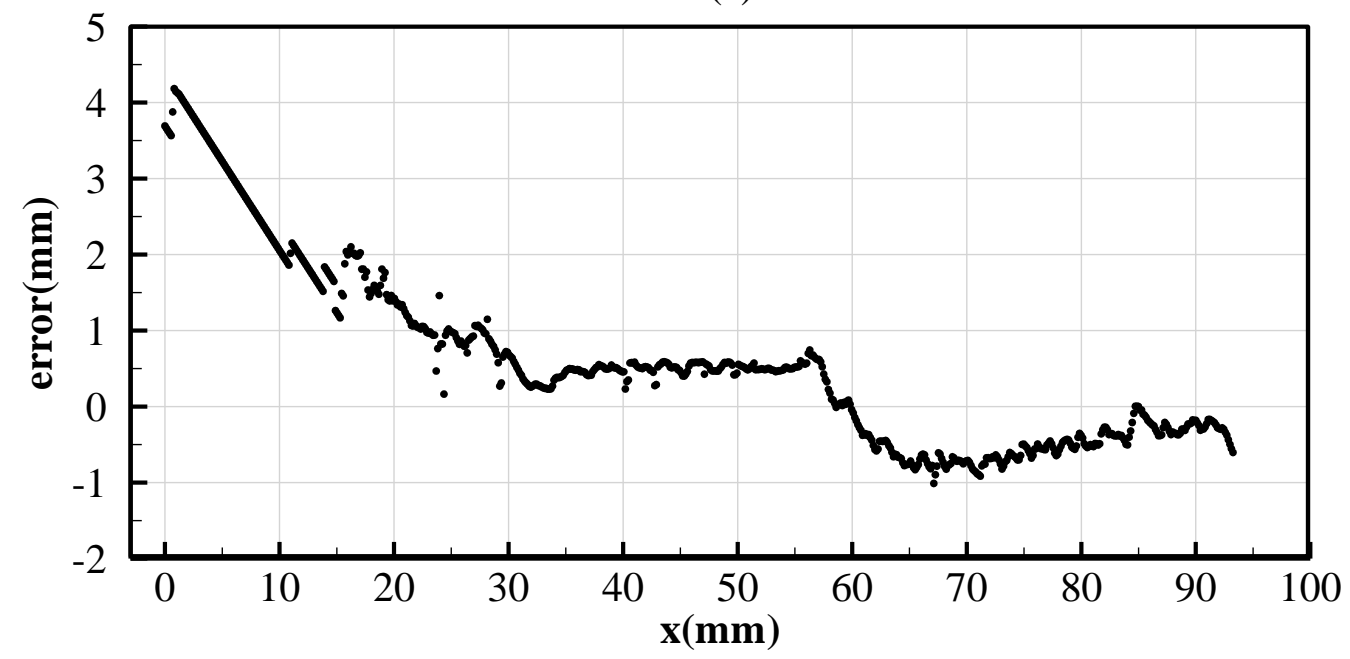

(b)

Figure 10 - (a) Estimated three-dimensional geometry (with dash line referring to the three cross sections in Figure 9 and (b) three-dimensional estimation error along the central plane $(y=30 \mathrm{~mm})$

After acquiring a depth value for each pixel, together with the pressure value obtained for each pixel in the second step, the three-dimensional pressure distribution over the model surface can be finally determined by using the pixel-to-pixel mapping. Figure 11 shows the three-dimensional 
pressure distribution and the Schlieren image for the flared cone model. The PSP results are averaged from ten instantaneous measurements, without post-processing.

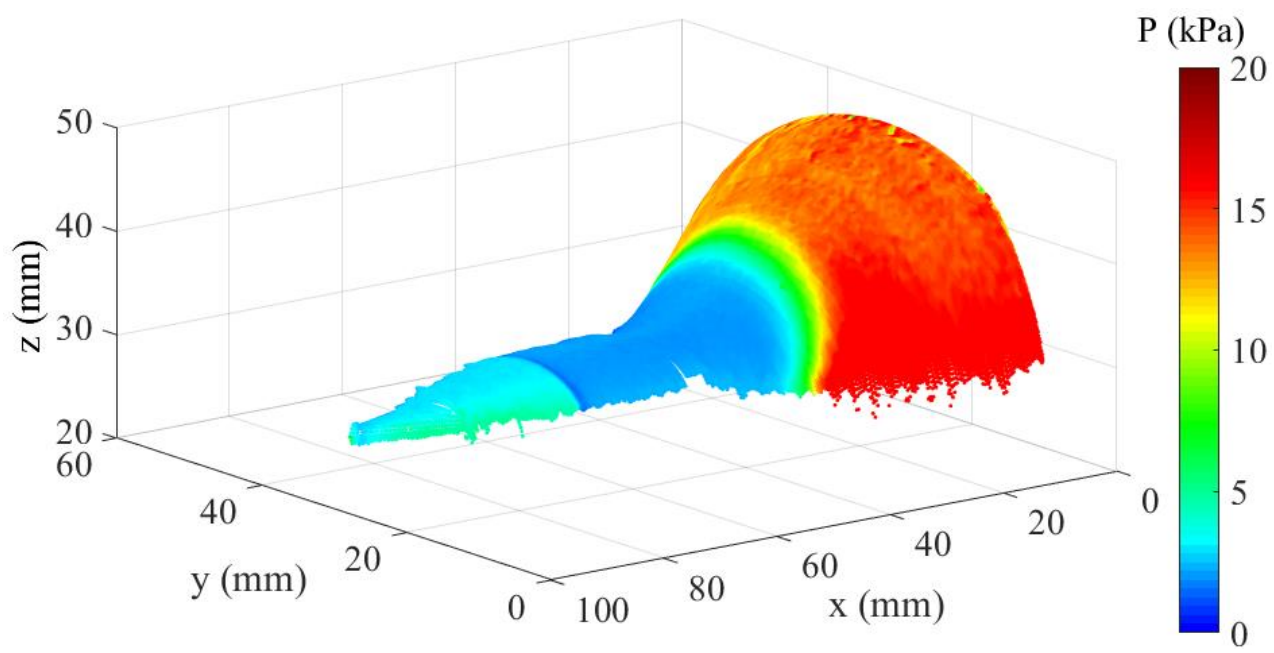

(a)

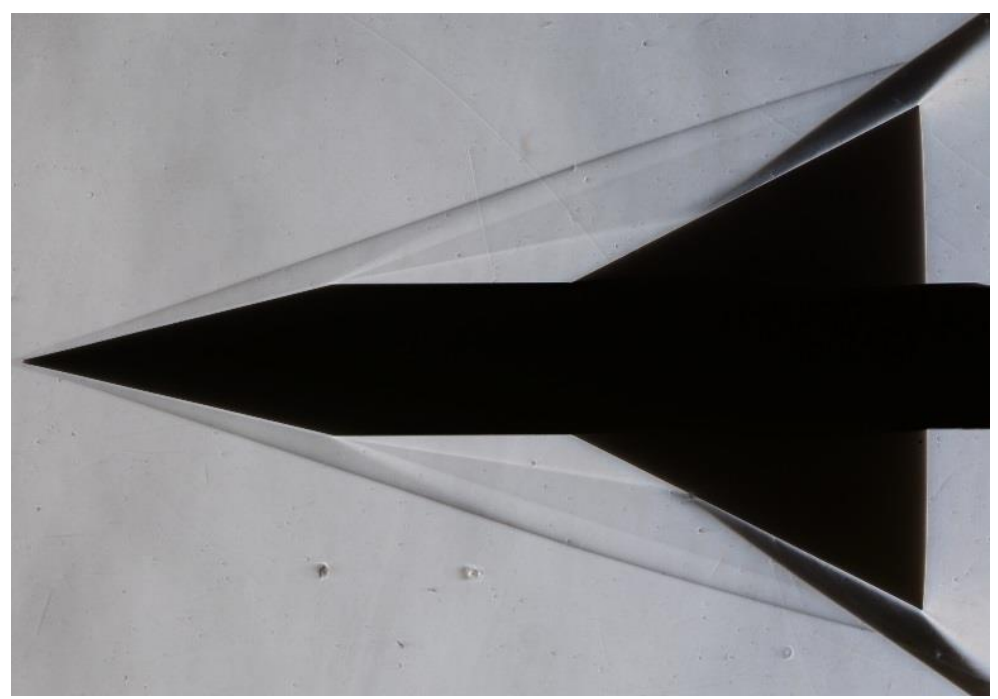

(b)

Figure 11 - (a) Measured three-dimensional surface pressure distribution for the flared cone model and (b) Schlieren image for the flared cone model

In order to allow a more quantitative investigation of the data, the scales were nondimensionalised as shown in Figure 12. The two-dimensional pressure image shown in Figure 12 (a) shows a slight asymmetry in the measured pressure profile, particularly around the leading 
part of the cone. During the run of the wind tunnel, there was slight sting deflection and model rotation, which effectively placed the model at a negative incidence of approximately 0.5 degrees (as estimated from the wind-on and -off schlieren images). This motion resulted in a slightly asymmetrical pressure distribution which is exacerbated in areas of the model with relatively large radii. It is noticeable near the trailing edge of the model that there are some nonaerodynamic features. These features are a result of the model rotation and unfortunately could not be corrected using image processing techniques. A schlieren image, flipped along the axis of the model, is overlaid with the original as shown in Figure 13. This figure (best viewed in colour), highlights that there are some slightly asymmetric flow features across the model. Asymmetries are most evident in the reattachment shock region.

The flow initially is compressed from the freestream static pressure to a higher value which then reduces to a value of $P / P_{\infty} \approx 3.4$ which is almost exactly equal to the theoretical value of a Mach 5 flow forced around a $14^{\circ}$ cone. From looking at the Schlieren image after this point the flow separates over the constant width section, something borne out by the PSP results showing constant pressure in this region. This is due to the large adverse pressure gradient from the compression corner of the flare causing the boundary layer to separate and propagate upstream until it reaches the expansion fan. The expansion fan turning angle is then reduced due to the separated flow (and subsequently formed shear layer) until the pressure is matched (noted by the straight shear layer). On the flared section of the cone the shear layer (from the separation region) impinges onto the flared surface creating a large, strong shockwave as seen by other authors (Erdem et al (2011b)). The pressure profile extracted in Figure 12 (b) is very similar to the CFD results calculated by Erdem (2011c) (Figure 4.19); however, the largest pressures recorded here are higher than predicted by CFD. Although one should never use CFD to validate an experiment, it is plausible that the PSP has over-predicted the pressure in this region as the highly compressed flow will be significantly hotter than other parts of the model, meaning that thermal quenching will take effect; manifesting as an increase in pressure. 


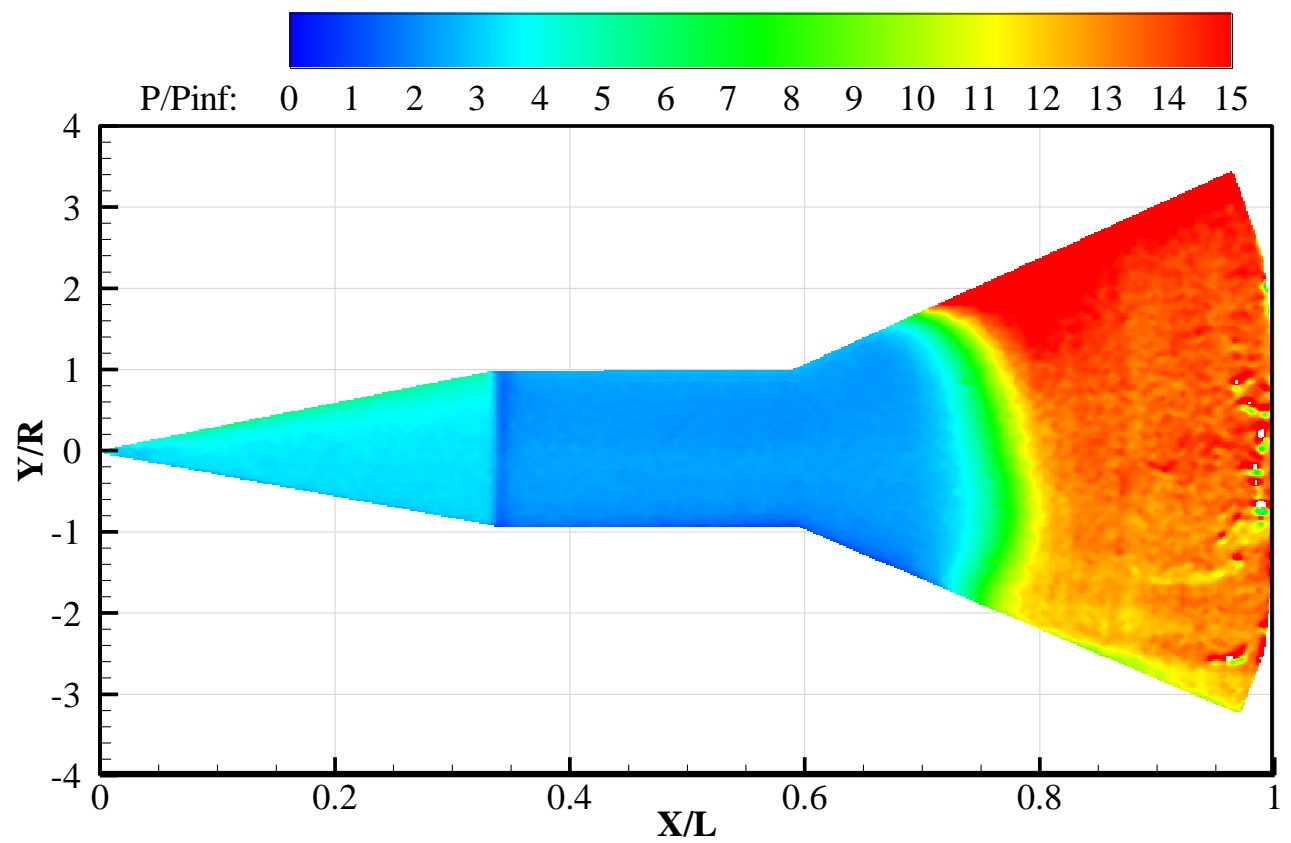

(a)

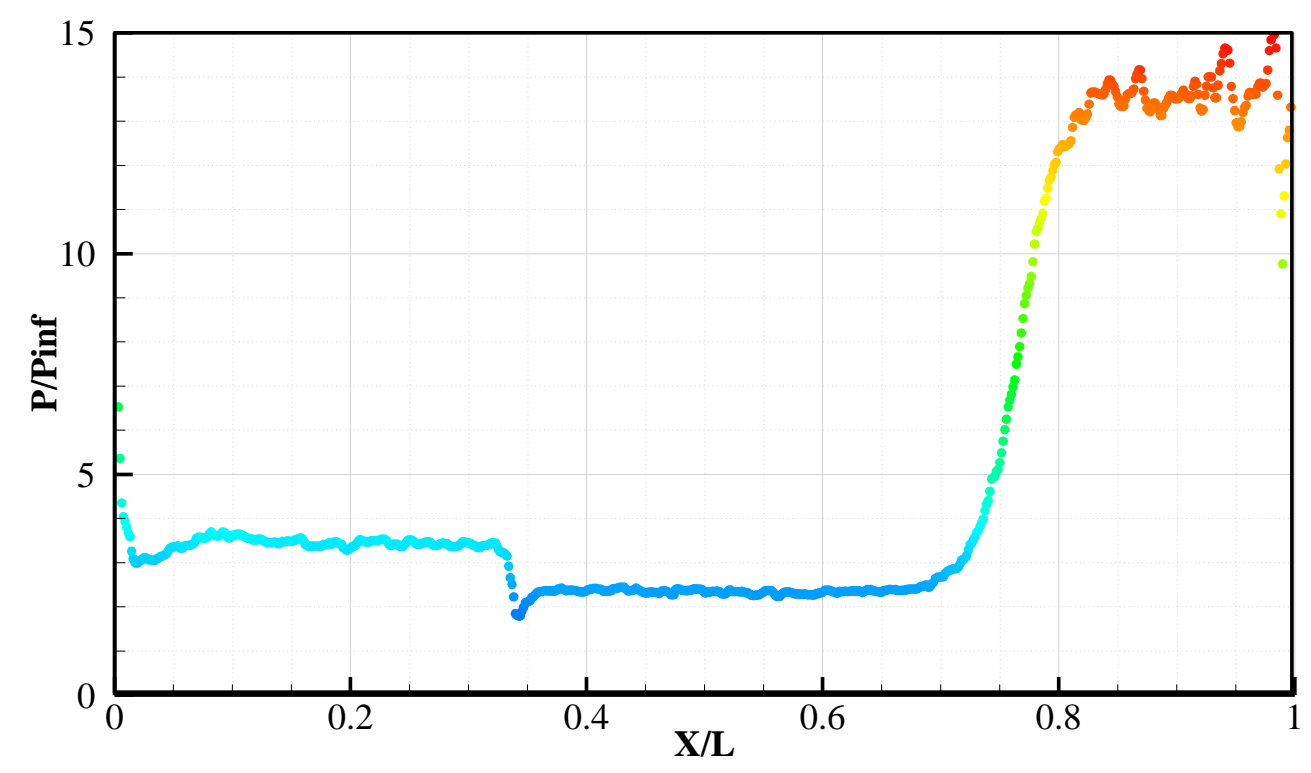

(b)

Figure 12 - (a) Two-dimensional pressure profile across the model and (b) the $Y / R=0$ profile extracted vs X/L 


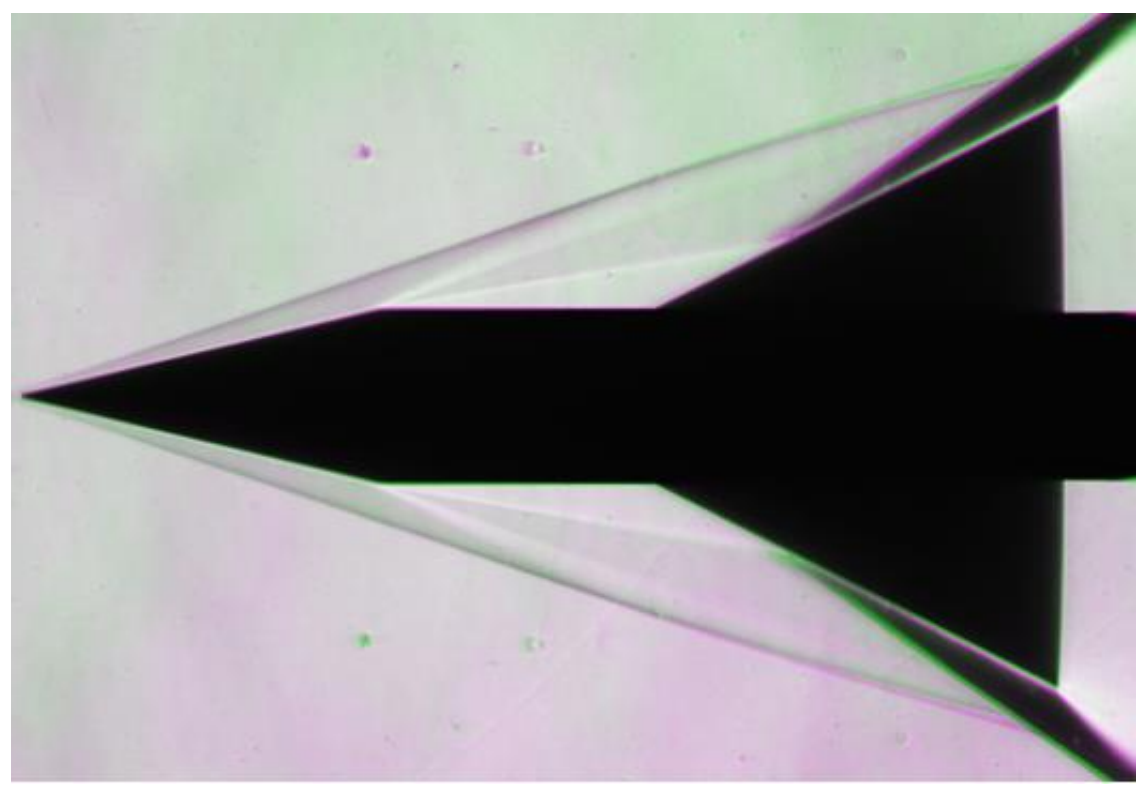

Figure 13 - A mirror of the schlieren image from Figure 11 (b) with the differences highlighted

\section{Conclusions}

This study presents a novel single-camera three-dimensional surface pressure measurement technique termed as light-field 3DPSP, which is achieved by combining single-camera light-field imaging and pressure-sensitive paint techniques. In the proposed light-field 3DPSP method, the wind-on and -off images are recorded by a high-resolution in-house-constructed light-field camera. A light-field rendering algorithm is used to generate the centre perspective for the windon and -off images, which are subsequently processed with traditional intensity-based method to obtain pressure information for each pixel. Limited by the current depth estimation method and lack of contrast on the model, a wind-off textured light-field image is necessary for calculating the depth information. The light-field 3DPSP technique was validated in a Mach 5 wind tunnel test by using a flared cone model and ISSI UniFIB PSP paint. The measured surface data agrees well with the design geometry and the recovered pressure information matches well with Schlieren results, despite some minor model motion. In conclusion, the light-field 3DPSP technique offers a very promising alternative for three-dimensional surface pressure measurement, especially for the space constraint applications, where mounting multiple cameras are difficult.

This study demonstrates the possibility for acquisition of model three-dimensional geometry and pressure data from a single camera system, especially for model with relatively small curvature 
surface which the current method could measure three-dimensional geometry with accuracy of micrometres. Alternatively, a projection system can be used after the wind-on data acquisition and during the wind-off acquisition to generate two separate meshes which can be aligned in regions of small model deflection. By using markers painted on the model surface and further improve the accuracy of light-field depth estimation algorithm, the model three-dimensional offset between wind-on and wind-off can be more accurately determined. This could be extended to taking lifetime-based PSP measurements (which are inherently less sensitive to temperature) and correcting for deformation of the model simultaneously. Two light-field 3DPSP measurements could be used to capture data around a whole model and their results can be stitched together to generate excellent-quality CFD validation data efficiently and with minimal wind tunnel utilisation.

\section{Acknowledgements}

Financial support provided by National Natural Science Foundation of China (Grant No. 11472175, 11772197) and Shanghai Raising Star Program (Grant No. 15QA1402400) are gratefully acknowledged.

\section{References}

Bell J, Schairer E, Hand L, Mehta, R (2001) Surface pressure measurements using luminescent coatings. Annu Rev Fluid Mech 33: 155-206

Ding J, Wang J, Liu Y, Shi S (2015) Dense Ray Tracing Based Reconstruction Algorithm for Lightfield Volumetric Particle Image Velocimetry. 7th Australian Conference on Laser Diagnostics in Fluid Mechanics and Combustion. Melbourne, Australia

Erdem E, Kontis K (2010) Numerical and experimental investigation of transverse injection flows. Shock Waves 20: 103-118

Engler R, Klein C, Merlo E, van Amerom P (2001) 360 degree PSP measurements in transonic flow. 19th International Congress on Instrumentation in Aerospace Simulation Facilities, Cleveland, Ohio, August 27-30 
Erdem E, Yang L, Kontis K (2011a) Drag Reduction Studies by Steady Energy Deposition at Mach 5. 49th AIAA Aerospace Sciences Meeting including the New Horizons Forum and Aerospace Exposition, Aerospace Sciences Meetings

Erdem E, Yang L, Kontis K (2011b) Experimental and numerical investigation of sharp/truncated cone-cylinder-flare Configurations at Mach 5 flow. International Journal of Aerospace Innovations

Erdem, E (2011c) Active Flow Control Studies at Mach 5: Measurement and Computation. University of Manchester PhD Thesis

Fahringer T, Lynch K, Thurow B (2015) Volumetric particle image velocimetry with a single plenoptic camera. Meas Sci Technol 26:115201

Gortler S, Grzeszczuk R, Szeliski R, Cohen M (1996) The lumigraph. ACM Trans Graph, 43-54

Georgiev T, Zheng K, Curless B, Salesin D, Nayar S, Intwala C (2006) Spatio-Angular Resolution Tradeoff in Integral Photography. Eurographics Symposium on Rendering

Gregory J, Asai K, Kameda M, Liu T, Sullivan J (2008) A review of pressure-sensitive paint for highspeed and unsteady aerodynamics. Proc IMechE, Part G: J Aerospace Engineering 222: 249-290

Gregory J, Sakaue H, Liu T, Sullivan J (2014). Fast pressure-sensitive paint for flow and acoustic diagnostics. Annual Review of Fluid Mechanics 46: 303-330

Jeon H, Park J, Choe G, Park J, Bok Y, Tai Y, Kweon I (2015) Accurate depth map estimation from a lenslet light field camera. In Proceedings of the IEEE Conference on Computer Vision and Pattern Recognition

Kavandi J, Callis J, Gouterman M, Khalil G, Wright D, Green E, Burns D, McLachlan B (1990) Luminescent barometry in wind tunnels. Rev Sci Instrum 61: 3340-3347

Levoy M, Hanrahan P (1996) Light field rendering. ACM Trans Graph, 31-42

Liu T, Sullivan J (2005) Pressure and temperature sensitive paints. Springer, Berlin.

Liu T, Burner AW, Jones TW, Barrows DA (2012) Photogrammetric techniques for aerospace applications. Progress in Aerospace Sciences, 54, 1-58

Levoy M (2006) Light fields and computational imaging. Computer 8: 46-55 
Li H, Ding J, Zhao Z, Qu W, Xiong J, Shi S. (2017) Investigation of 3D flow behaviour inside a 3×3 rod bundle using Light Field-PIV and the matched refractive index techniques. The 12th International Symposium on Particle Image Velocimetry. Busan, Korea

Ng R, Levoy M, Bredif M, Duval G, Horowitz M, Hanrahan P (2005) Light Field Photography with a Hand-Held Plenoptic Camera, tech. report CTSR 2005-02, Stanford University

Ng R (2006) Digital light field photography. PhD thesis, Stanford, CA, USA

Nabavi M (2010) Invited Review Article: Unsteady and pulsating pressure and temperature: A review of experimental techniques. Rev Sci Instrum 81: 031101

Quinn M K, Spinosa E, Roberts D A (2017) Miniaturisation of Pressure-Sensitive Paint Measurement Systems Using Low-Cost, Miniaturised Machine Vision Cameras. Sensors 17(8)

Shi S, Wang J, Ding J, Zhao Z, New TH (2016) Parametric study on light-field volumetric particle image velocimetry. Flow Meas Instrum 49: 70-88

Shi S, Ding J, New TH, Soria J (2017) Light-field camera-based 3D volumetric particle image velocimetry with dense ray tracing reconstruction technique. Exp Fluids 58:78

Vardaki E, Stokes N P, Patel S, Gustafsson, P (2012) Pressure Sensitive Paint Measurements on the Gripen Model at the ARA Transonic Wind Tunnel. 50th AIAA Aerospace Sciences Meeting including the New Horizons Forum and Aerospace Exposition

Xu S, Ding J, Zhao Z, Atkinson C, Soria J, Shi S. (2017) 3D flow measurements of circular air jet at $\operatorname{Re}=30,000$ using light field particle image velocimetry. The 12th International Symposium on Particle Image Velocimetry. Busan, Korea 